\title{
Provocative Tests for Myocardial Ischemia in Patients with Implantable Electronic Cardiac Devices
}

\author{
Gustavo Chiari Cabral11,*, Gabrielle Lima Pinto ${ }^{1}$, Andres Di Leoni Ferrari' ${ }^{1}$, Jessica Caroline \\ Feltrin Willes ${ }^{1}$, Paulo Ricardo Avancini Caramori ${ }^{1}$
}

\section{ORCID IDs}

Cabral GC (D) https://orcid.org/0000-0002-7992-8834

Willes JCF (D) https://orcid.org/0000-0002-0891-4427

Pinto GL (D) https://orcid.org/0000-0001-5822-9068

Caramori PRA (D) https://orcid.org/0000-0002-7895-8281

Ferrari AL (D) https://orcid.org/0000-0001-8975-7912

\begin{abstract}
Objective: Review the current literature in search of better knowledge about the use of implantable devices in patients who are investigating ischemic heart disease, and evaluate the possibility of its systematic use. Methods: Non-systematic literature review with exams and studies already published to date. Results: Risk stratification using implantable cardiac devices, associated with or as a substitute for the use of medication or physical stress, seems to be very safe, effective and highly reliable when compared to the methods traditionally established. Conclusion: Patients with implantable cardiac electronic devices constitute a specific challenging population, while research on ischemia is limited by secondary low/bay perfusion changes that result from the interaction of the device with the heart. These changes must be previously known, expected and interpreted with great care in order to reduce the number of false positives.
\end{abstract}

KEYWORDS: Ischemic heart disease; Pacemaker; Artificial cardiac stimulation; Ischemia.

\section{INTRODUCTION}

Increasingly present in the daily clinic, patients with implanted electronic cardiac devices (IECd), whether permanent pacemaker (PPM), implantable cardioverter defibrillator (ICD) or cardiac resynchronization therapy device (CRTd) may have clinical symptoms suggestive of myocardial ischemia. The increasingly sophisticated treatments, together with the advancement of drug treatments and the action of these devices in specific populations, allow greater patient survival and,

1. Pontíficia Universidade Católica - Hospital São Lucas - Serviço de Cardiologia - Porto Alegre/RS - Brazil. 
therefore, the appearance of concomitant cardiac disorders. This brings with it an increasing need for diagnostic evaluation and leads to the search for less aggressive and more accurate ways to perform them safely and effectively. The objective of this article is to review the literature in search of evidence to try to standardize the methods of assessing myocardial ischemia during the operation of these IECd during the myocardial stress phase, thus avoiding the use of medications and as a replacement tool physical stress due to an artificial increase in heart rate (HR) in patients with sinus node and/or atrioventricular disease.

\section{THEORETICAL FOUNDATION}

Certainly, ischemic disease is among the greatest fears and represents a huge portion of medical care in the most diverse centers. However, the investigation of ischemia in patients with IECd is still a field that needs observation and studies due to the changes, mainly mechanical and electrical, generated by its mode of operation.

Among the already established methods of investigating acute and/or chronic ischemic diseases, the indications and results remain irrefutable when the anatomical analysis with coronary angiography or coronary angiotomography are the methods employed. On the other hand, the search for ischemia using indirect methods still generates much discussion, since most of them are based on electrocardiographic and mechanical changes of the cardiac muscle, these being the main changes commonly found in patients with these devices.

\section{REVIEW}

\section{Exercise testing}

Exercise testing (ET) is useful in patients with IECd and should preferably be performed in a hospital environment in these cases. There are protocols proposed preferably for patients with devices, however, according to the guidelines aimed at performing the exam, these are not superior to the protocols used conventionally ${ }^{1}$.

To perform the exam safely, it is important to know the system to be evaluated (with or without an associated ICD), and the basic programming: minimum and maximum heart rate, if there is any type of $\mathrm{HR}$ response sensor programmed ("R mode"). The performance of ET in this context assists in the assessment of arrhythmias, effectiveness of drug treatment and determination of the patients' functional capacity, as well as investigating possible dysfunctions of devices, programming of biosensors to physical activity, allowing for better programming of these.

The sensors or biosensors correspond to a programmable tool of the device that allows the regulation of HR according to external stimuli or even the organism itself, through the perception of vertical and horizontal movements, vibrations and body temperature, changes in blood $\mathrm{pH}$, variation of blood QT, minute ventilation and blood volume in the right ventricle with variable specifications according to each manufacturer.

In the presence of IECd, the electrocardiogram (ECG) may or may not show spicules, depending on the HR demand of the patient at that moment, and which are presented as electrical artifacts resulting from the artificial electrical stimulus by the device. With technological developments, these have become increasingly subtle or even imperceptible to ECG (bipolar stimulation). Particularly in PPM, deflagration may be inhibited by the patient's intrinsic rhythm in those who exceed the programmed basic HR, or expected to be constant in dependent patients or with a CRT device. (Fig. 1).

Except in the presence of isolated atrial stimulation and intrinsic conduction for $100 \%$ of the time to the ventricles, allowing the evidence of native QRS (the presence of any artificial ventricular activation can trigger the phenomenon of electrical memory, altering the repolarization with inversion of the ECG T wave), the presence of aberrant QRS morphology is frequently observed. Depending on the type of device and the location of the ventricular electrode implantation, and also on the programming, they may present with a left or right branch block pattern, making it impossible to correctly evaluate 
the ST segment on the provocative ischemia test. Thus, in the presence of IECd, the analysis of ischemia becomes impaired due to secondary changes resulting from the QRS patterns described, being considered class III for its use by the Brazilian Society of Cardiology guidelines. ${ }^{2}$, with the exception of when it is associated with other methods with concomitant image evaluation or when the objective of the exam is to assess functional capacity or arrhythmia load.

Particularly in patients with ICD, attention should be paid to the possibility of triggering antiarrhythmic therapies during the increase in heart rate on examination. It is of utmost importance, therefore, to know the HR ranges and programmed therapies, in order to interrupt the effort 10 to 20 beats below these.

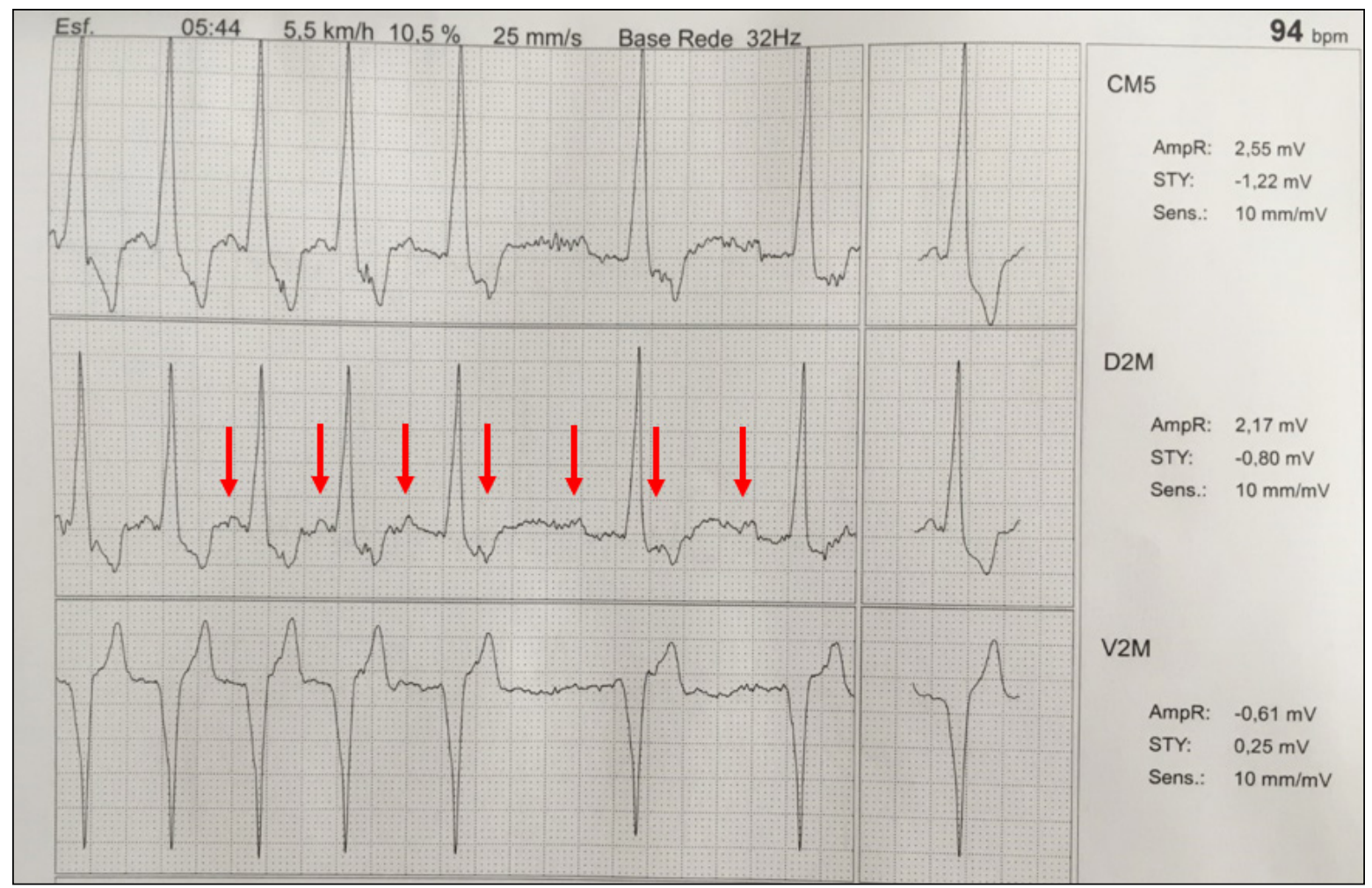

Figure 1. Exercise stress test for Investigation of myocardial ischemia in a patient with a pacemaker. Presence of 2: 1 conduction block with drop of the ventricular rate to $69-70 \mathrm{bpm}$ at the peak of exercise after reaching the maximum programmed cardiac frequency. Normal behavior considering the programming of the device.

\section{Magnetic resonance imaging}

It is estimated that approximately $70 \%$ of patients with IECd will have some magnetic resonance imaging (MRI) examination indicated at some point to assess their comorbidities. Cardiac MRI is an alternative in the assessment of myocardial ischemia in cases of unfavorable windows on echocardiography and low resolutions in nuclear medicine, although its use for investigating ischemia in patients with IECd, with pacemaker-induced stress, data are scarce. However, it integrates the assessment of global and segmental contractility, biventricular function, perfusion and myocardial viability, the quantification of the infarction area and does not use ionizing radiation or potentially nephrotoxic contrast. The pharmacological stress test can be performed with dobutamine and vasodilating agents (adenosine, dipyridamole and regadenoson).

Less than a decade ago, PPMs became compatible and safe for MRI in Brazil, and even less for ICD and CRT devices.

Surrounded by strict technical and monitoring specifications, and as long as they follow the guidelines recommended by the manufacturer, they are classified as conditional and non-conditional for MRI. The conditionals are those in which exposure to the magnetic field does not represent a risk to the patient, as long as the implanted system of cables and electrodes are from the same manufacturer. The rest are non-conditional MRI devices.

Some safety measures must be respected when examining patients with conditional IECD, such as monitoring heart rate and vital signs, the presence of qualified staff and equipment for immediate treatment of complications. Initially, the 
safety of performing MRI in patients with IECd was with exclusion zones, avoiding the chest, cervical region and upper abdomen, however, later it was safely authorized for the entire body. Some exclusion criteria must be respected, such as the presence of abandoned cables or electrodes, unconditional or epicardial electrodes, as well as implants for less than 6 weeks, non-thoracic devices and in children. These must be handled as non-conditional devices. In addition, patients with I C D must have antitachycardia therapies ensured and it is also recommended that the battery has a charge greater than $30 \%$.

In this way, as established by the Guidelines ${ }^{3}$, the stimulating physician has great importance in the routine evaluation of the patient in relation to the examination in this scenario. All devices must be evaluated before and after the MRI is performed to confirm the safety indication, the release for carrying out and the provision of data, such as: if the device is conditional; if there is the presence of the auto detect function ${ }^{3,4}$ or similar; if the programming will be done by a member of your team or if it is possible to perform the exam without the need for reprogramming, although it is emphasized that, in the current moment of the technology available in the country, most of them need to be reprogrammed for the asynchronous or similar mode, making the device indifferent to the magnetic field. In some cases of more modern devices, they are automatically programmed in safety mode when detecting the presence of an intense magnetic field, avoiding inappropriate shock in the case of the ICD, returning to the original programming right after the exam, thus avoiding the risk of competition from the asynchronously with the patient's intrinsic rhythm or with potential generation of symptomatic arrhythmias, such as pacemaker syndrome.

There are records of cases of MRI performed in patients with non-conditional devices without adverse effects, but their use has not yet been validated for routine use. If the examination is essential, some articles suggest that it be performed with reprogramming of the device, presence of the stimulator, with the appropriate safety parameters, lasting less than 40 minutes and a field less than or equal to $1.5 \mathrm{~T}$. It is important to point out that device dysfunction may occur, and temporary cardiac stimulation might be required.

\section{Echocardiography}

The echocardiogram allows global and segmental functional assessment of the left ventricle at rest or associated with stress, aiming at the investigation of myocardial ischemia. For patients with PPM, the non-invasive diagnosis of coronary artery disease is hampered by changes in the electrocardiographic analysis secondary to the artificial rhythm coming from the right ventricle. Stress in echocardiographic assessment becomes an effective option in these patients, allowing the analysis of systolic wall thickening and left ventricular wall movement, which can be influenced by ventricular stimulation. The changes in the ischemic myocardium begin with heterogeneous perfusion, metabolic changes, followed by diastolic dysfunction and regional systolic myocardial dysfunction preceding the ischemic changes in the ECG and, eventually, the angina clinic. This fact shows the diagnostic importance of the echocardiographic examination, mainly associated with the workload, which can be through exercise (preferable whenever possible), medication and through atrial stimulation or external programming of the IECd.

There have been reports since the 1980s on the performance of evaluation methods associated with stimulus by the PPM, be it definitive or just for the purpose of carrying out the exam, but without standardized protocols, absence of large comparative studies and some inconsistencies between the data in the literature. It was initially performed by transvenous pacemaker implantation and right atrial stimulation or transthoracic or transesophageal echocardiography methods combined with transesophageal atrial stress induced by stimulation of the left atrium through a catheter for nasal or oral transesophageal stimulation. There are variations of protocols referred to in the literature ${ }^{5-12}$ which consist of elevation of the stimulation HR in stages of 1 to 3 minutes until reaching the submaximal HR and, if tolerated, the maximum HR. Stress with atrial stimulation through transesophageal echocardiography allows HR to increase rapidly, seeking an ischemic response, with practically spontaneous recovery after the cessation of artificial stimulation, avoiding a prolonged state of ischemia. Atrial arrhythmogenicity is one of the possible side effects. Due to the semi-invasiveness of this method, its practical applicability is limited, despite being a test with good accuracy for detecting coronary artery disease.

More recently, the use of external PPM programming already implemented has been a possibility for carrying out the stress test, carried out through the controlled increase of the $\mathrm{HR}$ of stimulation until reaching the desired objective. It can 
be programmed externally at 100 to 110 impulses per minute, with increases of 10 impulses/minute every 2 to 3 minutes until $85 \%$ of the predicted maximum HR is reached or aiming at 150 impulses per minute, until the programmed maximum $\mathrm{HR}$ is reached for PPM or if limiting symptoms are triggered. Another protocol that could also be used and with a shorter exam duration is the stimulation at 100 impulses per minute for 3 minutes and $85 \%$ of the maximum HR expected for 3 to 5 minutes. It can also be performed in association with dobutamine. Atropine can be used in cases of second or third degree atrioventricular block (AVB), in a maximum dose of $2 \mathrm{mg}$, aiming at $1: 1$ conduction.

For all these scenarios, an external programmer and the participation of a stimulator are required. This method has good accuracy for identifying coronary artery disease and future events, being comparable to other stress echocardiogram methods ${ }^{13}$.It is safe, non-invasive, with good tolerance and allows HR to increase rapidly, seeking an ischemic response, with practically spontaneous recovery after the end of stimulation. It can be an alternative in patients with exercise intolerance, dobutamine use and in those with chronotropic incompetence.

Picano et al. ${ }^{7}$, in 2002, during a multicenter study with 46 patients, also with PPM previously implanted and with suspected or known coronary disease, submitted the patients to echocardiography with stress induced through the device. Regardless of the result, all patients were subsequently submitted to angiographic investigation. Fifteen patients were submitted to atrial stimulation and 31 to ventricular. 27 patients were considered positive for ischemic heart disease, showing a sensitivity of $70 \%$, specificity of $90 \%$ and accuracy of $78 \%$. Eight patients were considered false negative and two false positive. As expected, in a patient with ventricular stimulation, perfusion defects generally occur in the inferior and apical wall, reflecting the earlier activation resulting from the electrode positioning site.

Finally, some of these strategies presented must always be considered in patients with PPM undergoing investigation of myocardial ischemia, in view of their applicability and safety previously described.

\section{Scintigraphy}

Myocardial scintigraphy, less described in the literature in the context of association with IECd, was also effective. Presenting the same limitations as echocardiography, since, despite not directly assessing the motility of the myocardial walls, the mechanical alterations of the artificial stimulus also cause metabolic alterations resulting from abnormal cardiac activation, and determine perfusion defects - mainly in the inferior and apical wall. - which are often interpreted as changes in perfusion associated with potential ischemia. Another important fact to consider in patients undergoing chronic therapy by PPM is that the constant artificial myocardial activation by the device and all the desynchronization resulting from this functioning can lead to a picture of ventricular dysfunction and a drop in the ejection fraction, increasing the patients' morbidity and mortality, even without any correlation with the presence of ischemic disease.

The stimulation protocols proved to be quite safe. In general, small studies already carried out tend to prefer the atrial stimulus and to start the exam with a resting $\mathrm{HR}$ at 60-70 beats per minute, with HR progression every 1-3 minutes and an increase of 10-20 beats per stage, until reaching submaximal $\mathrm{HR}$, that is, $85 \%$ of the calculated maximum of the patient (age - $220 \mathrm{bpm}$ ), up to the upper limit allowed by the device or until adverse effects are referred to as signs of ventricular dysfunction, hypotension or severe hypertension, arrhythmias ventricular and/or clinical judgment. In some cases where atrioventricular blocks were evident, the administration of atropine was recommended in order to improve the conduction of the AV stimulus. The segmental analysis during the stress phase was also not performed uniformly in the studies, which hinders an adequate correlation and analysis among the published data.

Apparently, the stimulation by PPM suggests a good correlation, reliability and greater safety in relation to pharmacological stress, as it presents a shorter examination time, easier reversion to the patient's baseline status, absence of residual effects of medications and less care and guidance prior to exam. On the other hand, there is a need for trained staff and/or a doctor specialized in cardiac stimulation to be available to assist the exam, which is not a reality in many centers in our country.

Two studies performed cardiac catheterization systematically and compared the results found. In 2005, Cate et al. ${ }^{11}$ submitted 14 patients with normal angiography to myocardial scintigraphy with stress by right ventricular stimulation in patients with previously implanted PPM and with low doses of adenosine. 42 coronary territories were evaluated. Only three patients had myocardial perfusion completely normal at rest and $23(54.8 \%)$ of the 42 territories were considered 
abnormal, mainly in the lower, inferoseptal and apical regions. At the end of the study, only one patient maintained a normal coronary perfusion. In general, the changes found at rest, as expected due to ventricular stimulation, were in the inferior and apical wall, mainly close to the implantation site of the ventricular electrode. It is concluded, therefore, that perfusion defects close to the implantation site of the ventricular electrode must be carefully evaluated in these patients, as they may correspond to false positives in the absence of coronary disease.

\section{CONCLUSION}

According to this review, the use of IECd in the aid of risk stratification, associated or as a substitute for the use of medication or physical stress, seems to be very safe, effective and highly reliable in comparison to the methods already traditionally established. Secondary baseline perfusion changes and stimulation must be previously known, expected and interpreted with great care in order to reduce the number of false positives. Other points favorable to the non-pharmacological stimulus induced by PPM are the shorter examination time, easier return to the baseline parameters of the patient and the absence of residual effects of the medications, except for atropine, which may sometimes be necessary.

It is expected that, in the future, new studies with more patients and with all the different methods will be carried out, thus facilitating the standardization of protocols and a better understanding of the answers, bringing this practice more and more to the cardiologist's reality.

\section{ACKNOWLEDGMENT}

Not applicable.

\section{FUNDING}

Not applicable.

\section{AUTHORS ‘CONTRIBUTION}

Conceptualization: Cabral G. C. and Ferrari AL; Research: Cabral G. C., Pinto G. L. and Ferrari A. L .; Editorial Staff - Rascunho Original: Cabral G. C., Pinto G. L. and Ferrari A. L .; Writing - Reviewing and Editing: Cabral G. C., Pinto G. L., Ferrari A. L. and Willes J. C. F .; Resources: Cabral G. C., Pinto G. L., Ferrari A. L. and Willes J. C. F.; Supervision: Cabral G. C., Pinto G. L., Ferrari A. L., Willes J. C. F. and Caramori P. R. A.

\section{REFERENCES}

1. Hossri CAC, Felicioni SP, Lourenço UR, Silva PCC, Pachón El, Buglia S, et al. Teste Ergométrico em Portadores de Dispositivos Cardíacos Eletrônicos Implantáveis. Rev Bras Cardiol. 2014;27(3):217-27.

2. Meneghelo RS, Araújo CGS, Stein R, Mastrocolla LE, Albuquerque PF, Serra SM, et al. III Diretrizes da sociedade brasileira de cardiologia sobre teste ergométrico. Arq Bras Cardiol. 2010;95(5 Supl 1):1-26. https://doi.org/10.1590/S0066-782X2010002400001

3. Sara L, Szarf G, Tachibana A, Shiozaki AA, Villa AV, Oliveira AC, et al. Il Diretriz de Ressonância Magnética e Tomografia Computadorizada Cardiovascular da Sociedade Brasileira de Cardiologia e do Colégio Brasileiro de Radiologia. Arq Bras Cardiol. 2014;103(6 Supl 3):186. https://doi.org/10.5935/abc.20145006 
4. $\quad[\mathrm{ABCDI}]$ Associação Brasileira das Clínicas de Diagnóstico por Imagem. Orientações para realização de exames de ressonância magnética em portadores de dispositivos eletrônicos cardíacos. 2018. [citado em 5 out. 2020]. Disponível em: https://abcdi.org.br/ orientacoes-para-realizacao-de-exames-de-ressonancia-magnetica-em-portadores-de-dispositivos-eletronicos-cardiacos/

5. Płońska-Gościniak E, Gackowski A, Kukulski T, Kasprzak JD, Szyszka A, Braksator W, et al. Stress echocardiography. Part I: Stress echocardiography in coronary heart disease. J Ultrason. 2019;19:45-8. https://doi.org/10.15557/JoU.2019.0006

6. Biagini E, Schinkel AFL, Elhendy A, Bax J, Rizzello V, Van Domburg RT, et al. Pacemaker stress echocardiography predicts cardiac events in patients with permanent pacemaker. Am J Med. 2005;118(12):1381-6. https://doi.org/10.1016/j.amjmed.2005.04.040

7. Picano E, Alaimo A, Chubuchny V, Plonska E, Baldo V, Baldini U, et al. Noninvasive pacemaker stress echocardiography for diagnosis of coronary artery disease: A multicenter study. J Am Coll Cardiol. 2002;40(7):1305-10. https://doi.org/10.1016/S07351097(02)02157-5

8. Atar S, Nagai T, Cercek B, Naqvi TZ, Luo H, Siegel RJ. Pacing stress echocardiography: an alternative to pharmacologic stress testing. J Am Coll Cardiol. 2000;36(6):1935-41. https://doi.org/10.1016/S0735-1097(00)00964-5

9. Lee C-Y, Pellikka PA; McCully RB, Mahoney DW; Seward JB. Nonexercise stress transthoracic echocardiography: transesophageal atrial pacing versus dobutamine stress. J Am Coll Cardiol. 1999;33(2):506-11. https://doi.org/10.1016/S0735-1097(98)00599-3

10. Gligorova, Suzana; Agrusta, Marco. Pacing stress echocardiography. Cardiovasc Ultrasound. 2005;3:36. https://doi.org/10.1186/14767120-3-36

11. Cate TJFT; Visser FC; PAnhuyzen-Goedkoop NM; Verzijlbergen JF; Van Hemel NM. Pacemaker-related myocardial perfusion defects worsen during higher pacing rate and coronary flow augmentation. Heart Rhythm. 2005;2(10):1058-63. https://doi.org/10.1016/j. hrthm.2005.07.013

12. Nguyen TH, Horowitz JD, Unger SA. Tachycardic vs. pharmacologic stress myocardial perfusion imaging: differential implications in multi-vessel ischemia. Am J Cardiovasc Dis. 2012;2(1):34-42.

13. Pellikka PA; Nagueh SF.; Elhendy AA; Kuehl CA; Sawada SG. American Society of Echocardiography Recommendations for Performance, Interpretation, and Application of Stress Echocardiography. J Am Soc Echocardiogr. 2007;20(9):1021-41. https://doi. org/10.1016/j.echo.2007.07.003 